\title{
Velhice LGBT: uma análise das representações sociais entre idosos brasileiros
}

\section{La vejez LGBT: un análisis de las representaciones sociales entre los ancianos brasileños}

\section{LGBT old age: an analysis of the social representations among Brazilian elderly people}

\author{
Ana Gabriela Aguiar Trevia Salgado ${ }^{1}$ \\ Ludgleydson Fernandes de Araújo ${ }^{2}$ \\ José Victor De Oliveira Santos ${ }^{3}$ \\ Lorena Alves de Jesus ${ }^{4}$ \\ Luciana Kelly da Silva Fonseca ${ }^{5}$ \\ Daniel da Silva Sampaio ${ }^{6}$ \\ 1,2, 3, 4, 5, 6 Departamento de Psicología, Universidade Federal do Piauí. Brasil
}

\begin{abstract}
Resumo: Esta pesquisa tem como objetivo identificar as representações sociais da velhice LGBT entre a população idosa. Contou-se com a participação 100 pessoas, com idade entre 60 e 86 anos $(M=66,9$ anos; $D P=6,8)$. A maioria dos participantes declarou-se do sexo feminino $(69 \%)$, casados $(40 \%)$, católicos $(50 \%)$ e de orientação heterossexual (91,3\%). Foram utilizadas entrevistas semiestruturadas para a coleta de dados. Posteriormente, as entrevistas foram submetidas a uma análise, no software Iramuteq, pelo método da Classificação Hierárquica Descendente que originou 4 classes de aproximação semântica. Diante dos resultados, percebe-se que as Representações Sociais da velhice LGBT são, em sua maioria, carregadas de estigmas negativos e preconceito. Pretende-se, com esse estudo, contribuir para uma velhice bem-sucedida dessa parcela da população, a partir de uma discussão que propicie uma melhor compreensão do que é a velhice LGBT, bem como incentivar outros estudos a fim de orientar as práticas profissionais diante desse grupo.
\end{abstract}

Palavras chave: velhice, idosos, LGBT, sexualidade, representações sociais, Classificação Hierárquica Descendente

Resumen: Esta investigación tiene como objetivo identificar las representaciones sociales de la vejez LGBT entre la población anciana. Se contó con la participación de 100 personas, con edad entre 60 y 86 años $(M=66,9$ años, $D P=6,8)$. La mayoría de los participantes se declaró del sexo femenino $(69 \%)$, casados $(40 \%)$, católicos $(50 \%)$ y orientación heterosexual (91,3\%). Se utilizaron entrevistas semiestructuradas para la recolección de datos. Posteriormente, las entrevistas fueron sometidas a un análisis, en el software Iramuteq, por el método de la Clasificación Jerárquica Descendente que originó 4 clases de aproximación semántica. Ante los resultados, se percibe que las Representaciones Sociales de la vejez LGBT son, en su mayoría, cargadas de estigmas negativas y prejuicios. Se pretende, con este estudio, contribuir a una vejez exitosa de esa parcela de la población, a partir de una discusión que propicie una mejor comprensión de lo que es la vejez LGBT, así como fomentar otros estudios para orientar las prácticas profesionales frente a ese grupo.

Palabras clave: vejez, adultos mayores, LGBT, sexualidad, representaciones sociales, Clasificación Jerárquica Descendente

Abstract: This research aims to identify the social representations of LGBT old age among the elderly population. A number of 100 people participated in the research; they were aged between 60 and 86 years $(M=66.9$ years, $S D=6.8)$. Most participants were female $(69 \%)$, married $(40 \%)$, catholic $(50 \%)$ and heterosexual (91.3\%). Semi-structured interviews were used to collect data. Subsequently, the interviews were submitted to an analysis in the Iramuteq software by the Descending Hierarchical Classification method. It originated 4 classes of semantic approximation. Data obtained showed that the Social Representations of LGBT old age are mostly associated to negative stigma and prejudice. The purpose of this study is to contribute to a successful old age for this part of the population, based on a discussion that provides better understanding of what LGBT old age is, as well as encouraging other studies to guide professional practices for this group.

Key Words: old age, older adults, LGBT, sexuality, social representations, Descending Hierarchical Classification

Como citar este artigo:

Aguiar Trevia Salgado, A. G., Fernandes de Araújo, L., De Oliveira Santos, J. V., Alves de Jesus, L., da Silva Fonseca, L. K., \& da Silva Sampaio, D. (2017). Velhice LGBT: uma análise das representações sociais entre idosos brasileiros. Ciencias Psicológicas, 11(2), 155-163. doi: https://doi.org/10.22235/cp.v11i2.1487

Correspondência: Ana Gabriela Aguiar Trevia Salgado, Universidade Federal do Piauí, Campus de Parnaíba, Departamento de Psicologia. Av. São Sebastião, 2819. Cidade Universitária. 64202020 - Parnaíba, PI - Brasil, e-mail: gabrielatrevia@outlook.com. Ludgleydson Fernandes de Araújo, e-mail: ludgleydson@yahoo.com.br; José Victor De Oliveira Santos, e-mail: victorolintos@gmail.com; Lorena Alves de Jesus, e-mail: lorenaalve_s@hotmail.com._Luciana Kelly da Silva Fonseca, e-mail: l.kelly_fonseca@hotmail.com; Daniel da Silva Sampaio, e-mail: daniel99.sampaio@hotmail.com 


\section{Introdução}

O envelhecimento da população mundial é um fenômeno atual, de crescimento exponencial e que tem sido amplamente discutido em diversas áreas de conhecimento. Antes de mais nada, quando se aborda o tema envelhecimento populacional, trata-se de um fenômeno de transição geográfica, marcado pela inversão da pirâmide etária. Essa inversão é caracterizada pela diminuição da taxa de natalidade e aumento da longevidade, ocasionado pelas conquistas do conhecimento médico, urbanização adequada das cidades, melhoria nutricional, elevação dos níveis de higiene pessoal e ambiental, além de avanços em saúde como vacinas, uso de antibióticos e quimioterápicos que tornaram possível a prevenção ou cura de muitas doenças (Araújo \& Carvalho, 2005; Mendes, Gusmão Faro, \& Leite, 2005; Camarano, Kanso, \& Mello, 2004).

São considerados idosos o grupo de pessoas com faixa etária superior ou igual a 65 anos, nos países desenvolvidos, e 60 anos, nos países em desenvolvimento (Lei n. 10.741, 2003; Neri, 2008). De acordo com o Censo do Instituto Brasileiro de Geografia e Estatística ([IBGE], 2000), no ano do levantamento, $8,56 \%$ da população brasileira encontrava-se nessa faixa de idade. Em 2015, essa taxa aumentou para $14,3 \%$, de acordo com o Pesquisa Nacional por Amostra de Domicílios (IBGE, 2015). Estima-se que, em 2030, a população idosa represente $18,62 \%$ da população total brasileira. De acordo com o PNAD (2015), as regiões sul e sudeste do Brasil são as que possuem maior proporção de idosos, sendo $16 \%$ e $15,7 \%$, respectivamente.

Em consonância com a população total de idosos, supõe-se que a proporção de idosos LGBT também irá aumentar nas próximas décadas (A. C. F. Araújo, 2016; Kimmel, 2015). No Brasil, no Censo Demográfico de 2010, pela primeira vez foi identificada e enumerada a quantidade de relações homoafetivas. O IBGE (2010) identificou um total de 58 mil casais homoafetivos que vivem juntos no Brasil. Segundo Bergamo (2016, dezembro 5), recentemente, o presidente do Instituto, Paulo Rabello de Castro, declarou o interesse de fazer um levantamento da população LGBT no Brasil, o que possibilita um maior conhecimento acerca dessa parcela da população, bem como viabiliza maior atenção a essa categoria social.

Contudo, a ausência de conhecimento sobre essa população põe em risco o envelhecimento saudável dessas pessoas, cujas necessidades e experiências específicas permanecem, em grande parte, desconhecidas (Fernández-Rouco, Sánchez, \& González, 2012; Orel, 2014).

Em contrapartida ao que é mais amplamente divulgado, temos que a velhice também é carregada por aspectos positivos. Apesar das perdas no que concerne às capacidades físicas, sensoriais e neurológicas, a pessoa idosa pode apresentar ganhos relacionados às capacidades influenciadas pela cultura, inspirando o idoso a desenvolver-se nos domínios voltados às artes, lazer e do manejo das questões existenciais, visto que o idoso percorreu um longo caminho rico em experiências e vivências (Neri, 2008; Novaes, 1995).

Entre os diversos aspectos considerados no que concerne à qualidade vida, tem-se que a sexualidade é um aspecto primordial do ser humano e vai além do ato sexual, abrangendo identidades e papeis de gênero, erotismo, prazer, orientação sexual, intimidade e reprodução. Apesar de todas as dimensões onde a sexualidade pode estar incluída - pensamentos, atitudes, desejos, comportamentos, valores, entre outros -, nem sempre esta é vivenciada ou expressada. Desde a década de 1960, a sexualidade tem passado por um processo de individualização de comportamentos e normas. Dessa forma, além dos prismas biológicos e psicológicos, a sexualidade sofre influência de fatores sociais, econômicos, políticos, culturais, éticos, religiosos e espirituais (L. F. Araújo, 2016; Lima, Santiago, \& Arrais, 2014; Organização Mundial da Saúde [OMS], 2002; Santos, Carlos, Araújo, \& Negreiros, 2017).

A atual coorte de pessoas idosas LGBT viveu um período histórico onde não podiam compartilhar sua sexualidade, por medo de rejeição e perseguição, e tinham receio de admitir sua orientação para si mesmos, visto que haviam internalizado os estereótipos negativos que a sociedade impunha sobre a população LGBT (Fredriksen-Goldsen, Hoy-Ellis, Muraco, Goldsen \& Kim, 2015; Huyck, 1995 como citado em Papalia, 2013; Marques \& Sousa, 2016). Assim, muitos dos atuais idosos LGBT demoraram muitos anos para "sair do armário" (Scherrer \& Fedor, 2015).

De acordo com Facchini e França (2009), o termo LGBT refere-se às lésbicas, gays, bissexuais, intersexuais, onde o $\mathrm{T}$, além de representar a presença de travestis e transexuais, em alguns locais no Brasil, diz respeito também à transgêneros, isto é, pessoas que não se identificam com o comportamento ou papel esperado do seu sexo biológico determinado pelo nascimento, a exemplo: 
crossdressers, drag queens, transformistas e outros.

Tendo em vista o cenário que a comunidade LGBT enfrenta por conta do preconceito histórico que traz consigo uma série de violências motivadas pelos estereótipos negativos, discriminação e intolerância; um contexto social caracterizado pelo processo de discriminação que vai desde o extermínio direto com requintes de crueldade até manifestações de intolerância no trabalho, na família, na religião, na escola, no atendimento médico, nos meios de comunicação e na população em geral, percebe-se que as pessoas idosas que fazem parte dessa categoria social também sofrem com esses estigmas.

Embora as pesquisas sobre a velhice estejam emergindo entre os temas investigados pelas Ciências Sociais, sobretudo pela Psicologia, explorar e analisar o processo de envelhecimento no contexto da orientação sexual do idoso ainda é pouco articulado. Tendo em vista que no Brasil comporta-se uma série de dificuldades e desafios para abordar o tema, a luta por visibilidade é um ato constante na vida dessas pessoas (Santos, Carlos, Araújo, \& Negreiros, 2017; Wladirson \& Chaves, 2012).

Contudo, à medida que ocorre o aumento da população idosa e a população LGBT tem o reconhecimento de seus direitos firmado na sociedade, é necessário um aprofundamento no conhecimento sobre as pessoas LGBT mais velhas (Berger, 1982 como citado em Marques \& Sousa, 2016; Berger \& Kelly, 2002 como citado em Marques \& Sousa, 2016; Minichiello, Plummer \& Loxton, 2004 como citado em Marques \& Sousa, 2016).

Sob uma visão dinâmica, as representações sociais configuram-se como uma rede de conceitos e imagens interativas desenvolvidas pelos indivíduos e grupos, cujos conteúdos evoluem continuamente através do tempo e do espaço, sendo esse processo cada vez mais intenso conforme a complexidade e velocidade das comunicações disponíveis (Carvalho \& Arruda, 2008; Jodelet, 1989; Morera, Padilha, Silva, \& Sapag, 2015; Moscovici, 1984; Sêga, 2000). Os conceitos e imagens formados a partir das representações sociais são maneiras de interpretar a realidade cotidiana. Jodelet $(1989 ; 2016)$ caracteriza a representação como uma forma de conhecimento prático que conecta um sujeito a um objeto, ou seja, ela estabelece um vínculo entre um sujeito, individual ou social, e o objeto que ela substitui.

As representações sociais permitem a compreensão do mundo, a partir de um objetivo prático, onde são construídos e compartilhados um grupo de conhecimentos, entendimentos, conceitos, explicação sobre alguém, algum objeto ou algum fato. Dessa forma, as representações sociais orientam as atitudes das pessoas frente ao objeto da representação. Estudar a percepção da sociedade sobre a velhice e o envelhecimento LGBT permite a identificação das concepções que as pessoas têm sobre esses eventos.

Nesse contexto, buscou-se, através da Teoria das Representações Sociais, identificar e comparar as representações sociais da velhice LGBT entre a população idosa brasileira.

\section{Método}

\section{Tipo da investigação}

Trata-se de um estudo descritivo e exploratório com dados transversais.

\section{Participantes}

A pesquisa contou com a participação 100 (cem) pessoas idosas brasileiras, de ambos os sexos, com idade entre 60 e 86 anos $(\mathrm{M}=66,9$ anos; $\mathrm{DP}=6,8)$. A maioria dos participantes declarou-se do sexo feminino (69\%), casados (40\%), católicos (50\%), de orientação heterossexual $(91,3 \%)$, com renda familiar média de 1800 reais $(23,8 \%)$, cursaram até o ensino médio $(25,6 \%)$ e aposentados (70,7\%). Os participantes são residentes dos estados brasileiros do Piauí, Ceará e Pará.

\section{Instrumentos}

Para a coleta de dados foram utilizados dois instrumentos. O primeiro foi um questionário sóciodemográfico para caracterização dos participantes, com a finalidade de obter informações sobre idade, sexo, estado civil, renda, orientação sexual, religião, estado em que vive, se tem algum parentesco com algum indivíduo com orientação sexual homossexual e se convive com algum idoso LGBT; o segundo foi uma entrevista semiestruturada, para compreender as percepções dos participantes sobre velhice e homossexualidade LGBT, com a seguinte questão norteadora: "Como você entende a velhice LGBT?".

\section{Procedimentos éticos e de coleta de dados}

A presente pesquisa foi submetida ao Conselho de Ética em Pesquisa - CEP da Universidade 
Federal do Piauí (Brasil), apresentando CAEE: 57225916.1.0000.5214 e número do parecer: 1.755.790. Após aprovação pelo Comitê, foi iniciada a coleta de dados de forma voluntária e anônima, onde foram esclarecidos os objetivos do estudo e obtidas as devidas autorizações e o preenchimento dos Termos de Consentimento Livre e Esclarecido, para que os participantes pudessem autorizar sua participação na pesquisa e responder aos instrumentos, como determina a resolução 466/12 do Conselho Nacional de Saúde brasileiro (CNS). Estima-se que aproximadamente 30 minutos foram necessários para finalizar a participação na pesquisa.

Utilizou-se o critério estabelecido por $\mathrm{Ca}$ margo (2005), que indica a partir de 20 unidades de contexto inicial, para definição do número de participantes necessários para a pesquisa. Nessa investigação, foram colhidos 100 questionários, sendo uma parte da amostra por conveniência, onde os participantes foram abordados em locais públicos como praças e shoppings e outra parte escolhida de forma não-probabilística, intencional e acidental em grupos de convivência de idosos. A coleta dos dados foi realizada nos estados brasileiros do Ceará, Pará e Piauí.

\section{Análise de dados}

Os dados sócio-demográficos foram analisados a partir das estatísticas descritivas no software SPSS for Windows versão 21, objetivando caracterizar a amostra.

As entrevistas semiestruturadas foram submetidas a uma Classificação Hierárquica Descendente (CHD) simples com o auxílio do software Iramuteq versão 0.7. De acordo com Camargo (2005), esse tipo de análise permite a obtenção de classes lexicais, caracterizadas por vocábulos específicos e pelos segmentos de texto que possuem esses vocábulos em comum.

\section{Resultados}

A análise dos dados, no software Iramuteq, constituída de 100 entrevistas, ou unidades de contexto iniciais (u.c.i.), resultou em 80 unidades de contexto elementar (u.c.e.), que deram origem ao dendograma (figura 1). Foram formadas 4 classes de aproximação semântica, porém não foi possível estabelecer um perfil de pessoas em cada classe. A primeira partição dividiu o Corpus em dois sub-corpus, separando a classe 4 das demais.

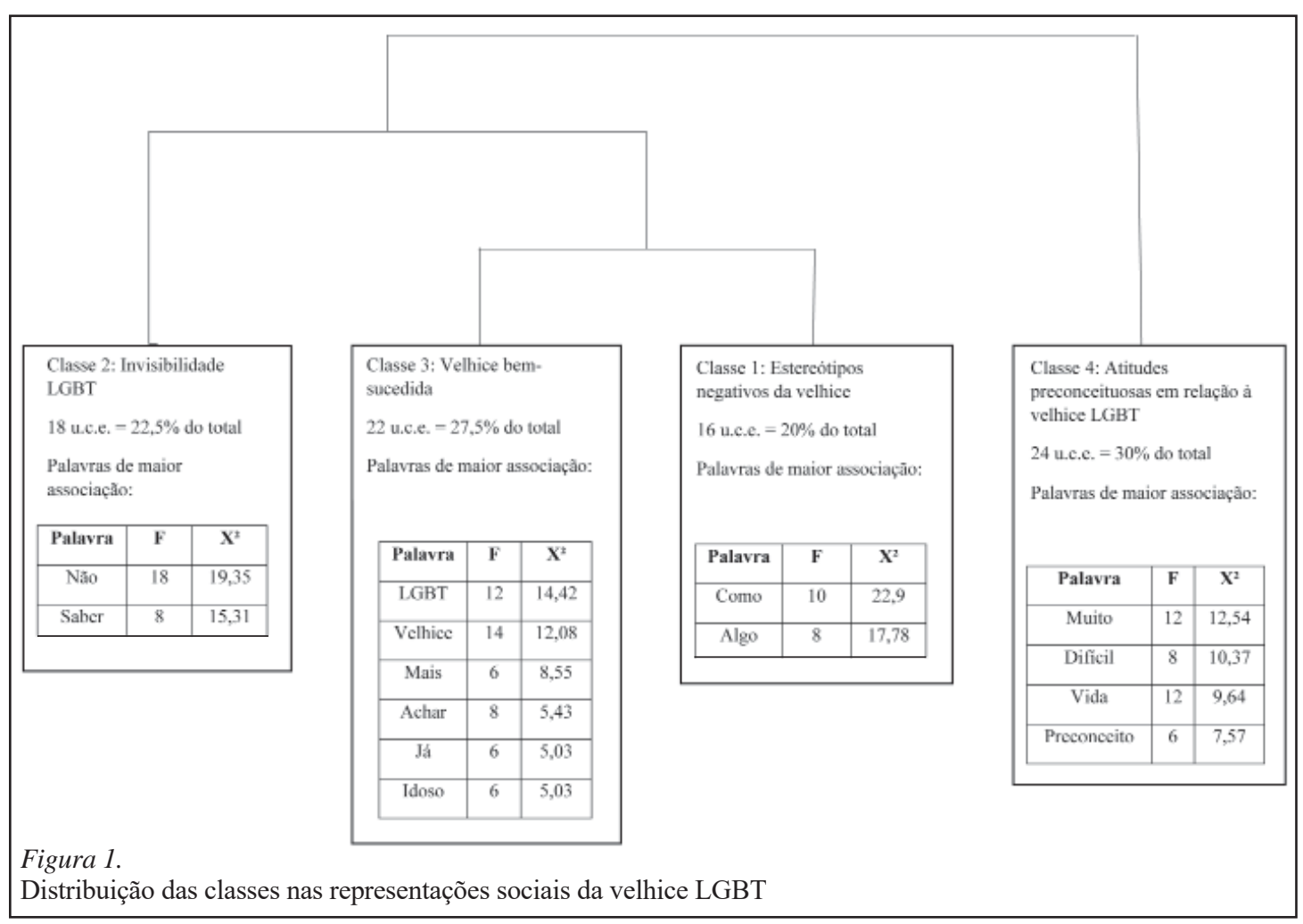


A segunda partição fragmentou o sub-corpus maior, originando a classe 2 . Aúltima partição deu origem às classes 1 e 3 .

A classe 1 é a menor das classes, constituída por 16 u.c.e., representando $20 \%$ do total de unidades. Essa classe foi denominada Estereótipos negativos da velhice. Predomina, nessa classe, a ideia de que a velhice LGBT é como qualquer velhice. Contudo, são enfatizados alguns aspectos negativos da velhice, como declínio de habilidades, abandono e necessidade de maior cuidado em virtude de doenças. Além disso, nessa classe são ainda encontrados alguns sentimentos negativos frente aos idosos LGBT, como "pena de quem é homossexual", haja vista que o idoso que segue uma orientação diferente da heterossexual sofre com os estigmas da velhice e da orientação sexual.

A classe 2 é composta por $22,5 \%$ do total de u.c.e. (18 unidades). Levando em consideração/o as variáveis descritivas, pode-se caracterizar essa classe como sendo constituída, em sua maior parte, por idosos do sexo masculino (10 u.c.e.). Nessa classe, percebe-se a predominância de discursos onde os idosos declaram não saber opinar ou não ter conhecimento acerca dos idosos LGBT. Por esse motivo, denominou-se essa classe Invisibilidade LGBT.

A terceira classe, constituída de 22 u.c.e (27,5\% do total), tem como principais conteúdos que a velhice LGBT deve ser como qualquer velhice. Observa-se que os discursos dessa classe afirmam que o idoso LGBT afirma a sua orientação ainda na juventude, sendo esse um dos aspectos que determina uma "boa velhice". Em contrapartida ao que foi encontrado na classe 1 , tem-se nessa classe uma perspectiva positiva do envelhecimento: paz, tranquilidade, estabilidade e maturidade. Denominou-se essa classe Velhice bem-sucedida.

A classe 4 constitui-se como a maior classe (24 u.c.e. $-30 \%$ das unidades). Denominada Atitudes preconceituosas em relação a velhice LGBT, a quarta classe exprime conteúdos relacionados à hostilidade frente à velhice LGBT. As compreensões acerca da velhice LGBT nessa classe são de uma fase da vida marcada pelo preconceito e desprezo da sociedade, sendo solitária, complicada e conturbada.

\section{Discussão}

A partir da observação e interpretação dos dados obtidos na pesquisa, percebe-se, de modo geral, que muitos estereótipos do envelhecimento e da velhice LGBT apresentados em estudos ante- riores são confirmados. Assim, serão discutidos os conteúdos lexicais que estruturam o dendograma dentro de cada classe identificada.

\section{Classe 1 - Estereótipos negativos da velhice}

Os conteúdos obtidos na classe Estereótipos negativos da velhice pouco diferenciam a velhice heterossexual da velhice LGBT, com discursos que afirmam que esta é "como qualquer velhice". Entretanto, essa afirmação vem associada aos estigmas da velhice como uma fase da vida solitária, onde os idosos sofrem com o abandono e o desprezo de familiares e da sociedade de um modo geral, além do comprometimento de algumas habilidades físicas e psíquicas. Ressalta-se que esses dados fazem oposição aos resultados identificados em pesquisas anteriores que apresentam as representações sociais dos idosos em relação à velhice.

Os discursos que mais representam essa classe são "acredito que tende para muitas dificuldades e sofrimento, solidão"; "algo difícil"; "é como a velhice de quem é heterossexual, uma época de declínio das habilidades psíquicas e motoras e necessidade de um cuidado maior por parte da família"; "algo preocupante se levado em consideração o modo como são tratados os idosos no nosso país pois não existem ações que priorizem a gente e sendo de uma condição sexual diferente agrava sua situação enquanto sujeito estigmatizado duplamente".

Frequentemente, as pessoas não idosas representam a velhice e o envelhecimento de forma negativa. Essas diferenças significativas de percepções justificam-se porque grupos têm a tendência de representar socialmente outros grupos de forma diferente, principalmente quando esses grupos são de gerações diferentes (Araújo \& Carvalho, 2005; Camargo, Contarello, Wachelke, Morais, \& Piccolo, 2014; Cruz \& Assunção Ferreira, 2011; Daniel, Antunes, \& Amaral, 2015).

Contudo, apesar de concordar em alguns aspectos, essas representações divergem da concepção geral dos idosos que tendem a representar a velhice de forma mais positiva, acreditando que as experiências que tiveram ao longo da vida até o momento produziram sabedoria e as relações sociais, o trabalho e o sentimento de utilidade complementam a ideia de que a velhice é uma fase exitosa, principalmente as relações familiares que se tornam centrais em suas vidas (Camargo et. al., 2014; Comerlato, Guimarães, \& Alves, 2009). 
No contexto social, a velhice é comumente apresentada de forma desvalorizada. Essa imagem negativa e estereotipada que é disseminada faz com quem muitas pessoas que chegam à velhice assumas essas características (Araújo \& Carvalho, 2005; Oliveira et. al., 2012). Assim, é natural que alguns idosos representem a velhice como sinônimo de doença, declínio e morte (Costa \& Campos, 2009; Dias, Paúl, \& Watanabe, 2014; Guerra \& Caldas, 2010; Vianna, Loureiro, \& Alves, 2012).

Além disso, algumas pessoas, ainda que já tenham idade superior a 60 anos, não se percebem como velhos e, por terem preservadas as habilidades cognitivas, sociais, emocionais e físicas, consideram que a condição de idoso é uma realidade distante (Passamani, 2013; Santos, Carlos, Araújo \& Negreiros, 2017). Assim, a negação da velhice também é uma possível justificativa para as representações identificadas nessa classe.

\section{Classe 2 - Invisibilidade LGBT}

Nessa classe, verifica-se a presença de discursos que retratam o quanto a população idosa LGBT é encoberta, ignorada, esquecida e desprezada pela sociedade. Essa classe ficou marcada por expressões como "não sei opinar"; "não conheço"; "não ouvi falar"; "solidão, mas não entendo muito do assunto"; "é um comportamento que causa dificuldade de compreensão"; "não sei explicar mas acho que é triste e solitária"; "nunca pensei no assunto".

Haja vista os estigmas acerca da sexualidade na velhice, falar de homossexualidade, bissexualidade ou transsexualidade nessa fase da vida torna-se ainda mais dificultoso, principalmente porque as pessoas idosas LGBT ainda são vítimas de invisibilidade na sociedade (Adams, 2015 como citado em Araújo \& Fernández-Rouco, 2016; Persson, 2009 como citado em Araújo \& Fernández-Rouco, 2016; Santos, Carlos, Araújo \& Negreiros, 2017), como fica exposto pela análise dos conteúdos lexicais.

A invisibilidade dos idosos LGBT possui algumas possíveis justificativas: em primeiro lugar, pela tendência da sociedade em estereotipar os idosos como "assexuados" e pela concepção de muitos psicogerontólogos de que quase todos os adultos mais velhos são heterossexuais e os que não seguiam essa orientação já eram muito idosos para seguir outra orientação; em segundo lugar, pela dificuldade de estudar essa população que, muitas vezes, evita exposição por medo de serem vítimas de preconceito; em terceiro lugar, pelo interesse da Geriatria e da Gerontologia em estudar outros aspectos do envelhecimento, como as doenças crônicas. Dessa forma, as necessidades e a existência dos idosos LGBT foram por muito tempo ignoradas pela maioria das instituições e pela sociedade (Dorman et al., 1995 como citado em Orel, 2014; Kimmel, Hinrichs \& Fisher, 2015; Quam \& Whitford, 1992 como citado em Orel, 2014).

\section{Classe 3 - Velhice bem-sucedida}

Os conteúdos constatados na classe 3 fazem referência a um ponto de vista da velhice como uma etapa proveitosa da vida. Discursos como "vida cômoda e confortável"; "um momento de liberdade da escolha acerca das realizações sexuais e afetivas"; "plenitude"; "deve ser feliz"; "entendo que foi uma conquista na vida do seu direito [de se revelar LGBT]"; "nesse caso assume importância tão grande que a pessoa quer é ser feliz e devemos respeitar"; "nunca é tarde para ser feliz"; "com paz e tranquila" caracterizam essa classe.

Como citado anteriormente, os idosos, principalmente os que fazem parte de grupos de convivência, tendem a simbolizar a velhice como um momento prazeroso, onde tornam-se protagonistas de sua vida, já que os filhos estão crescidos e os idosos se aposentaram do trabalho (Camargo et. al., 2014; Comerlato, Guimarães \& Alves, 2009). Reconhecer a velhice LGBT como um período feliz e pleno significa assumir que esses idosos possuem também a garantia de viver essa fase usufruindo dos benefícios e direitos conquistados ao longo da vida.

Por outro lado, acreditar que os idosos LGBT possuem iguais condições de vivenciar a velhice como os idosos heterossexuais denota desconsiderar as disparidades desses dois públicos. Muitos idosos LGBT vivem em situação de vulnerabilidade, por exemplo, no que diz respeito à saúde. Os idosos LGBT possuem necessidades particulares, especialmente no caso de idosos transexuais (Fernández-Rouco, Sánchez, \& González, 2012) que têm mais dificuldade de acesso aos dispositivos de atenção à saúde e experimentam mais disparidades de atenção à saúde, com maior sofrimento psíquico e menor saúde física de modo 
geral (Fredriksen-Goldsen et. al., 2015; Scherrer $\&$ Fedor, 2015).

Cahill (2015) apresenta algumas iniciativas que já foram adotadas nos Estados Unidos e outras que podem ser adotadas para oferecer melhor qualidade de vida às pessoas idosas LGBT. Entre essas iniciativas estão o melhor preparo dos profissionais que vão atender essa população, a elaboração de uma lei que designe os idosos LGBT como uma população com maior necessidade social, promoção de educação em saúde sexual, serviços de saúde mental e programas de suporte de renda.

Assim, é importante perceber que é fundamental lançar um olhar diferenciado sobre a população LGBT no que diz respeito às necessidades específicas desse grupo, para que, dessa forma, possa ser oferecida, de fato, uma velhice bem-sucedida para esses idosos.

\section{Classe 4 - Atitudes preconceituosas em relação a velhice $L G B T$}

Essa classe é a que mais se discrimina das demais, sendo caracterizada por discursos que retratam que a velhice LGBT é carregada por dificuldades em virtude do preconceito enraizado na sociedade. Percebe-se que nas falas dos participantes é revelada a presença de fortes estereótipos acerca dessa parcela da população, todavia os idosos participantes da pesquisa não se reconheciam enquanto parte dessa sociedade que trata as pessoas idosas LGBT com preconceito.

Alguns conteúdos como "errado, mas é uma escolha da pessoa"; "acho feio porque é muito estranho, mas não tenho nada contra"; "nunca vi de perto mas é escolha de cada um"; "no seio da sociedade, essa relação, acredito, é puro sofrimento"; "é muito difícil principalmente pelo preconceito"; "parece uma vida complicada"; "acredito que tende para muitas dificuldades $e$ sofrimento por causa do preconceito que as pessoas têm"; "acho que na velhice LGBT o sofrimento é grande pelo desprezo da sociedade"; "difícil, qualquer parte do ciclo LGBT é conturbada com a hipocrisia social"; "acho que é difícil ser homossexual em qualquer fase da vida"; "uma superação depois de enfrentar muito preconceito ao longo da vida"; "penso que o desprezo das pessoas é muito grande por causa do preconceito".

Além de se eximir da responsabilidade de praticar também o preconceito contra a pessoa idosa LGBT, observa-se que os participantes da pesquisa demonstram traços de preconceito sutil em seus discursos. Diferente do preconceito flagrante, o preconceito sutil é indireto, distante e "frio", sendo uma forma mais velada ou disfarçada de exprimir a opinião preconceituosa (Allport, 1954; Lima \& Vala, 2004).

\section{Conclusão}

A presente pesquisa versou sobre as representações sociais da velhice LGBT entre a população idosa em três estados brasileiros: Piauí, Ceará e Pará. Os dados obtidos possibilitaram a apreensão de representações sistematizadas em quatro classes.

Verifica-se que essas representações se relacionam, em grande parte, com a forma como os idosos participantes da pesquisa vivenciam a sua própria velhice. Os idosos que percebem e vivem essa fase como algo negativo ou não se reconhecem como idosos sustentam a noção de que a velhice LGBT é igualmente conturbada e desafiadora, sendo essa concepção acentuada, principalmente, em virtude dos estereótipos existentes acerca das pessoas LGBT; já os que a vivenciam de forma agradável e prazerosa representam a velhice LGBT também desse modo, ainda que preterindo as discrepâncias e particularidades desses grupos.

Ademais, a invisibilidade e o preconceito identificados nas representações identificadas sugerem a necessidade de produção e difusão dos conhecimentos acerca desse grupo de pessoas, que se mostrou desassistido e desprezado na população entrevistada.

Assim, espera-se que essa pesquisa possa encorajar outros estudos a respeito do tema, bem como orientar as práticas dos profissionais da gerontologia com os idosos, a fim de dissolver os estereótipos negativos dessa fase da vida.

\section{Referências}

Allport, G.W. (1954). The nature of prejudice. Reading: Massachusetts.Addison-Wesley Publishing Company.

Araújo, A. C. F. (2016). Rompendo o silêncio: desvelando a sexualidade em idosos. UNILUS Ensino $e$ Pesquisa, 12(29), 34-41. Recuperado de: http://revista. unilus.edu.br/index.php/ruep/article/view/689

Araújo, L. F. (2016). Aspectos Psicossociais da Velhice LGBT. Psicologia em Estudo, 21(2), 359-361.

Araújo, L. F., \& Carvalho, V. Â. M. D. L. (2005). Aspectos sócio-históricos e psicológicos da velhice. Mneme Revista de Humanidades, 6(13), 228-236. Recuperado de: https://periodicos.ufrn.br/mneme/article/view/278 
Araújo, L. F., \& Fernández-Rouco, N. (2016). Idosos LGBT: Fatores de Risco e Proteção. Em Falcão, D. V. da S., Pedroso, J. da S., \& Araújo, L. F. de (orgs.). Velhices: temas emergentes nos contextos psicossocial e familiar (pp. 129-138). Campinas: Alínea.

Bergamo, M. (2016, dezembro 5). IBGE pretende fazer primeira pesquisa nacional sobre população LGBT. Folha de São Paulo. Recuperado de: http://www1.folha.uol.com.br/colunas/monicabergamo/2016/12/1838027-ibge-pretende-fazer-primeira-pesquisa-nacional-sobre-populacao-lgbt.shtml

Cahill, S. (2015). Community resources and government services for lgbt older adults and their families. In N. A. Orel \& C. A. Fruhauf (Orgs.). The lives of LGBT older adults: Understanding challenges and resilience (pp. 141-169). Washington, DC: American Psychological Association.

Camarano, A. A., Kanso, S., \& Mello, J. L. (2004). Como vive o idoso brasileiro. Em Camarano, A. A. Muito além dos 60 (pp. 25-73). Rio de Janeiro: IPEA.

Camargo, B. V. (2005). ALCESTE: Um programa informático de análise quantitativa de dados textuais. Em A. S. P. Moreira; J. C. Jesuíno \& B. V. Camargo (Org.). Perspectivas teórico-metodológicas em representações sociais (pp. 511-539). EdUFPB: João Pessoa.

Camargo, B. V., Contarello, A., Wachelke, J. F. R., Morais, D. X., \& Piccolo, C. (2014). Representações sociais do envelhecimento entre diferentes gerações no Brasil e na Itália. Psicologia em Pesquisa, 8(2), 179-188. doi: https://dx.doi.org/10.5327/Z1982-1247201400020007

Carvalho, J. G. S., \& Arruda, A. (2008). Teoria das representações sociais e história: um diálogo necessário. Paidéia, 18(41), 445-456. doi: https://dx.doi.org/10.1590/ S0103-863X2008000300003

Comerlato, E. M. B., Guimarães, I., \& Alves, E. D. (2009). Tempo de plantar e tempo de colher: as representações sociais de profissionais de saúde e idosos sobre o processo de envelhecimento. Revista Eletrônica de Enfermagem, 9(3). doi: https://doi.org/10.5216/ree. v9i3.7481

Costa, F. G., \& Campos, P. H. F. (2009). Representação social da velhice, exclusão e práticas institucionais. Revista Eletrônica de Psicologia e Políticas Públicas, 1(1), 100-113. Acesso através do site do CRP-09 www. crp09.org.br

Cruz, R. C., \& Assunção Ferreira, M. (2011). Um certo jeito de ser velho: representações sociais da velhice por familiares de idosos. Texto \& Contexto Enfermagem, 1, 144-151. doi: https://dx.doi.org/10.1590/ S0104-07072011000100017

Daniel, F., Antunes, A., \& Amaral, I. (2015). Representações sociais da velhice. Análise Psicológica, 33(3), 291301. doi: https://dx.doi.org/10.14417/ap.972

Dias, M., Paúl, C., \& Watanabe, H. (2014). Representações sociais de velhice e suas relações com declínio e finitude em comentários e críticas publicados na mídia. Revista Kairós: Gerontologia, 17(1), 125-143. Recuperado de: https://revistas.pucsp.br/index.php/ kairos/article/view/20286

Facchini, R., \& França, I. L. (2009). De cores e matizes: sujeitos, conexões e desafios no Movimento LGBT brasileiro. Sexualidad, Salud y Sociedad-Revista Latinoamericana, (3), 54-81. Recuperado de: http:// www.e-publicacoes.uerj.br/index.php/SexualidadSaludySociedad/article/view/41/466

Fernández-Rouco, N., Sánchez, F. L., \& González, R. J. C. (2012). Transexualidad y vejez: una realidad por conocer. Kairós Gerontologia. Revista da Faculdade de Ciências Humanas e Saúde, 15(3), 15-25. Recuperado de: https://revistas.pucsp.br/index.php/kairos/article/ view/15308/11442

Fredriksen-Goldsen, K. I., Hoy-Ellis, C. P., Muraco A., Goldsen, J., \& Kim, H. J. (2015). The health and well-being of lgbt older adults: disparities, risks, and resilience across the life course. In N. A. Orel \& C. A. Fruhauf (Orgs.). The lives of LGBT older adults: Understanding challenges and resilience (pp. 25-53). Washington, DC: American Psychological Association.

Guerra, A. C. L. C., \& Caldas, C. P. (2010). Dificuldades e recompensas no processo de envelhecimento: a percepção do sujeito idoso. Ciência \& Saúde Coletiva, 15(6), 2931-2940. https://dx.doi.org/10.1590/ S1413-81232010000600031

Instituto Brasileiro de Estatística (IBGE) (2000). Censo Demográfico 2000. Rio de Janeiro: IBGE.

Instituto Brasileiro de Estatística (IBGE) (2010). Censo Demográfico 2010. Rio de Janeiro: IBGE.

Instituto Brasileiro de Estatística (IBGE) (2015). Pesquisa nacional por amostra de domicílios. Rio de Janeiro: IBGE.

Kimmel, D. C. (2015). Theories of aging applied to LGBT older adults and their families. In N. A. Orel \& C. A. Fruhauf (Orgs.). The lives of LGBT older adults: Understanding challenges and resilience (pp. 73-90). Washington, DC: American Psychological Association.

Kimmel, D. C., Hinrichs, K. L., \& Fisher, L. D. (2015). Understanding lesbian, gay, bisexual, and transgender older adults. In Peter A. Lichtenberg, Benjamin T. Mast, Brian D. Carpenter, Julie Loebach Wetherell (Eds.), APA handbook of clinical geropsychology, Vol. 1: History and status of the field and perspectives on aging (pp. 459-472). Washington, DC, US: American Psychological Association.

Jodelet, D. (1989). Representações Sociais: fenômeno, conceito e teoria. Paris: Presses Universitares de France.

Jodelet, D. (2016). A representação: noção transversal, ferramenta da transdisciplinaridade. Cadernos de Pesquisa, 46(162), 1258-1271. doi: https://dx.doi. org/10.1590/198053143845

Lei no 10.741 , de $1^{\circ}$ de outubro de 2003. Dispõe sobre a Política Nacional do Idoso, e dá outras providências. Diário Oficial da União, Brasília, 3.

Lima, A. A., Santiago, K. C., \& Arrais, A. R. (2014). Homossexualidade: Sexualidade no envelhecimento. Temporalis, 2(28), 221-239. doi: https://doi. org/10.22422/2238-1856.2014v14n28p221-239

Lima, M. E. O., \& Vala, J. (2004). As novas formas de expressão do preconceito e do racismo. Estudos de Psicologia (Natal), 9(3), 401-411. doi: https://dx.doi. org/10.1590/S1413-294X2004000300002

Marques, F. D., \& Sousa, L. (2016). Portuguese Older Gay Men: Pathways to Family Integrity. Paidéia (Ribeirão Preto), 26(64), 149-159. doi: https://dx.doi. org/10.1590/1982-43272664201602

Mendes, M. R., Gusmão, J. L. D., Faro, A. C. M., \& Leite, R. D. C. B. D. (2005). A situação social do idoso no Brasil: uma breve consideração. Acta paulista de enfermagem, 18(4), 422-426. doi: https://dx.doi. org/10.1590/S0103-21002005000400011

Morera, J. A. C., Padilha, M. I., Silva, D. G. V. da, \& Sapag, J. (2015). Theoretical and methodological aspects of social representations. Texto \& Contexto - Enfermagem, 24(4), 1157-1165. doi: https://dx.doi. org/10.1590/0104-0707201500003440014

Moscovici, S. (1984) The phenomenon of social representations. Em, R.M. Farr e S. Moscovici (Orgs.) Social Representations. Cambridge: Cambridge University Press. 
Neri, A. L. (2008). Palavras-chave em gerontologia. 2a ed. Campinas: Alínea.

Novaes, M. H. (1995). Psicologia da terceira idade: conquistas possíveis e rupturas necessárias. Grypho.

Oliveira, A. M. M., Lopes, M. E. L., Evangelista, C. B., de Oliveira, A. E. C., Gouvéia, E. M. L., \& Duarte, M. C. S. (2012). Representações sociais e envelhecimento: uma revisão integrativa de literatura. Revista Brasileira de Ciências da Saúde, 16(3), 427-434. doi: https:// dx.doi.org/10.4034/RBCS.2012.16.03.21

Orel, N. A. (2014). Investigar as Necessidades e Preocupações dos Adultos Idosos, Gays, Bissexuais e Transgêneros: o uso de metodologia qualitativa e quantitativa. Journal of Homosexuality , 61 (1), 5378. doi: http://doi.org/10.1080/00918369.2013.835236

Organização Mundial da Saúde (2002). Etienne G. Krug, Linda L. Dahlberg, James A. Mercy, Anthony B. Zwi, \& Rafael Lozano (eds.). Relatório mundial sobre violência e saúde. Genebra: Organização Mundial da Saúde.

Papalia, D. E., \& Feldman, R. D. (2013). Desenvolvimento humano. 12.ed. Porto Alegre: Artmed.

Passamani, G. R. (2013, setembro). Velhice, homossexualidades e memória: notas de campo no Pantanal sul-matogrossense. Seminário Internacional Fazendo Gênero (Anais Eletrônicos), Florianópolis, SC, Brasil, 10. Recuperado de: http://www.fg2013.wwc2017. eventos.dype.com.br/resources/anais/20/1381508577 ARQUIVO_GuilhermeRPassamani.pdf
Santos, J. V. O.; Carlos, K. P. T.; Araújo, L. F. \& Negreiros, F. (2017). Compreendendo a velhice LGBT: uma revisão da literatura. In: L. F. Araújo; C. M. R. G. Carvalho. (Org.). Envelhecimento e Práticas Gerontológicas (pp. 81-96). 1ed. Curitiba-PR/Teresina-PI: Editora CRV/EDUFPI.

Scherrer, K. S. \& Fedor, J. P. (2015). Family issues for LGBT older adults. In N. A. Orel \& C. A. Fruhauf (Orgs.). The lives of LGBT older adults: Understanding challenges and resilience (pp. 171-192). Washington, DC: American Psychological Association.

Sêga, R. A. (2000). O conceito de representação social nas obras de Denise Jodelet e Serge Moscovici. Anos 90, 8(13): 128-133. Disponível em: http://seer.ufrgs.br/ index.php/anos90/article/view/6719/4026

Vianna, L. G., Loureiro, A. M. L., \& Alves, V. P. (2012). O velho e a morte. Revista Kairós: Gerontologia, 15, 117-132. Disponível em: https://revistas.pucsp.br/ index.php/kairos/article/view/17040

Wladirson, C., \& Chaves, E. (2012). Entretecendo diálogo entre homossexualidade e velhice: notas analítico-interpretativas acerca do envelhecimento gay. Revista do NUFEN, 4(1), 34-43. Disponível em: http://pepsic.bvsalud.org/scielo.php? script $=$ sci arttext\&pid $=$ S2175$-25912012000100004 \& \operatorname{lng}=$ pt\&tlng=pt. 